\title{
Anaesthetic Management in Peripartum Cardiomyopathy
}

\author{
Gamunu Ratnayake ${ }^{1}$ and Vinod Patil ${ }^{2 *}$ \\ ${ }^{1}$ Specialist Registrar Anaesthetics, Kings College Hospital, UK \\ ${ }^{2}$ Consultant Anaesthetist, Queens Hospital, UK
}

Submission: February 28, 2018; Published: March 20, 2018

*Corresponding author: Vinod Patil, Consultant Anaesthetist, Queens Hospital, Romford, London, England, UK, Email: vinodpatil@doctors.org.uk

\begin{abstract}
Peripartum cardiomyopathy (PPCM) is a rare condition associated with significant morbidity and mortality. It requires careful anaesthetic management to ensure both mother and child are safely supported through to delivery. The use of neuroaxial anaesthetics must be balanced with their haemodynamic effects and the patient's ability to compensate for such physiological strains. This review outlines the epidemiology, management and outcomes associated with PPCM. Special consideration is made for the anaesthetic implications in the pre-operative, operative and post-operative periods for patients requiring caesarean section.
\end{abstract}

Keywords: Peripartum cardiomyopathy; Heart failure; Congestive cardiac failure; Third trimester; Inotropes; Anaesthetic management

\section{Introduction}

Peripartum cardiomyopathy (PPCM) is a form of dilated cardiomyopathy which presents with the signs and symptoms of heart failure late in the third trimester of pregnancy, up to 5 months postpartum [1]. For a diagnosis of PPCM, there should be no other cause for cardiac failure such as viral illness or ischaemic heart disease. The echocardiographic criteria include an Ejection Fraction $(\mathrm{EF})<45 \%$, fractional shortening $<30 \%$ with an end diastolic dimension of $>2.7 \mathrm{~cm} / \mathrm{m} 2$ [2]. Although PPCM is a rare disease, affecting $0.1 \%$ of all pregnancies, it has a morbidity and mortality rate of up to one third [3,4]. Sliwa et al. [5] looked at the incidence of PPCM in 2010. They reported a relatively equal distribution between the Caucasian and black populations. This is in contrast to previous studies which indicated a greater incidence in black patients, especially from Africa [5].

The main associations with PPCM were multiparity $(>3$ children), twin pregnancies and preeclampsia (and to a lesser extent other hypertensive diseases) [5,6]. Maternal age (>30 years old) was also a significant risk factor [7]. Infection with Human Immunodeficiency Virus (HIV) seems to have a strong association with PPCM, especially in patients outside of the European Union [5]. An exact aetiology has not been found for this heterogenous condition. This is likely to be due to its multifactorial nature, with a definition that is confined more to a specific time period for diagnosis than a specific pathophysiology. The underlying mechanism seems to be oxidative stress with decreased angiogenesis [1]. Oxidative stress maybe perpetuated by hypertension, abnormal haemodynamic stress response, potential viral aetiology and nutritional deficiency [8]. There may be an immunologically mediated mechanism with sensitisation of maternal antibodies to foetal cells. These may sensitise maternal antibodies to myocardial epitopes, which would explain the presence of antibodies to cardiac myosin heavy chains in some PPCM patients $[9,10]$. Prolactin metabolism may also contribute to the disturbance of cardiomyocyte angiogenesis leading to cardiac failure [11].

\section{Discussion}

Given the rare nature of this disorder and the complicating factor of pregnancy there are no clinical trials to inform the optimum management of these patients. The treatment is that of acute severe decompensated cardiac failure. This involves protection of the airway and maintenance of oxygenation with positive pressure ventilation. Loop diuretics are safe in the peripartum period and used to diurese patients [1]. Both the preload and afterload are reduced with continuous infusions of nitrates such as glyceryl-trintrate (GTN) or sodium nitroprusside (which needs to be used carefully due to the production of cyanide molecules). Some patients may require inotropic support which can either be pharmacological (such as Milrinone or dobutamine) or mechanical (left ventricular assist devices, aortic balloon pumps or veno-arterial extracorporeal membrane oxygenation) [12]. 
It is important to recognize the increased thromboembolic incidence in patients with an ejection fraction of $<35 \%$. They can form left ventricular thrombi, and so should be on low molecular weight heparin whilst pregnant and warfarin following delivery [13]. The haemodynamic strain placed on the mother during pregnancy is well recognised. With the increased intravascular volume, compression of pelvic veins, increased placental vascular bed and blood requirements of the gravid uterus, cardiac failure can be exacerbated. There is an argument for early delivery of the baby. This is somewhat less problematic for the foetus since this condition manifests in the last month of pregnancy so the foetus should be viable [13]. In patients with refractory PPCM, there is some evidence for women to stop breast feeding their children and starting them on Bromocriptine to reduce serum prolactin levels. Stopping breastfeeding and treatment with bromocriptine for even 1 week improves morbidity and mortality [14]. This study found a $20 \%$ improvement in left ventricular systolic function after a week. It should however be noted that no placebo was used in this study, rather it was compared to another study where the drug was not given to the patients.

The data regarding prolactin blockade is somewhat difficult to interpret as a study surveying mothers with PPCM who breast fed, found that those that did breast feed had a better chance of recovering left ventricular function [15]. Once patients are stabilised during their acute presentation, they should be started on routine heart failure medications. This includes beta blockers, digoxin, loop diuretics and hydralazine prior to delivery of the baby. In the postpartum period, the choice of medications depends on the mother's desire to breastfeed. Angiotensin converting enzyme inhibitors (ACEi) or angiotensin receptor antagonists should be avoided in breastfeeding mothers as it can cause profound neonatal hypotension $[12,16]$. The outcomes from PPCM have improved dramatically as more intensive treatment modalities and careful monitoring of PPCM patients has evolved. Six month mortality from PPCM has reduced to around 3\% [1]. Half of patients will recover their normal cardiac function within 5 years [17]. However up to $4 \%$ of patients may require heart transplant [6].

\section{Anaesthetic Considerations}

The anaesthetic management of these patients can be challenging, given their reduced physiological reserve and potential detrimental effects on the baby. Any patient presenting with PPCM will almost certainly be reviewed by an anaesthetist and may require emergency interventions. The anaesthetic considerations can broadly be divided into pre-operative, operative and post-operative for caesarean sections. Even if the woman will undergo a vaginal (non-operative) delivery the same operative considerations are important.

\section{Pre-operative considerations}

The signs and symptoms of PPCM can be subtle initially and be mistaken for normal changes in pregnancy such as leg swelling, shortness of breath, orthopnoea and paroxysmal nocturnal dyspnoea. These patients can have a first presentation of acute pulmonary oedema and decompensated heart failure. A standard airway, breathing and circulation (ABC) approach should be employed. If intubation is required, care must be taken as with any pregnant woman about awareness, maintenance of perfusion pressure to the placenta and risk of difficult airway. Bridging with non-invasive positive pressure (continuous positive pressure (CPAP) or rarely pressure support ventilation) is usually effective and obviates the need for intubation unless patients are in extremis. The management is primarily diuresis with oral and intravenous loop diuretics and vasodilation with nitrates to reduce pre and afterload. If these measures fail, inotropes and mechanical assist devices could be used.

It is important when managing these patients in the acute setting to consider a wide differential diagnosis, as PPCM is essentially a diagnosis of exclusion. Conditions such as an acute ischaemic event, aortic dissection, viral myocarditis and renal pathologies such as renal artery stenosis should be ruled out. Immediate investigations should include an ECG (to look for ischaemia), blood tests including troponin and Brain Naturetic peptide (nt-BNP) and renal function, chest x-ray (to rule out chest infections) and echocardiogram. Urgent further investigations would include cardiac catheterization to look for ischaemic heart disease and cardiac MRI to look for infiltrative disease and an accurate ejection fraction. Other investigations can include cardiac biopsies. An HIV test is important and should be repeated in any patient presenting with a cardiomyopathy in pregnancy (despite a negative test at booking).

These patients may need optimisation with inotropic agents such as dobutamine or milrinone. These should ideally be started with cardiac output monitoring. Patients should be managed in a high dependency or intensive care setting, with continuous ECG, saturation and invasive blood pressure monitoring. As with any complicated obstetric patient, a multidisciplinary approach with involvement from consultant midwives, obstetricians, anaesthetists, critical care doctors and cardiologists is vital. Daily review by specialist cardiology teams is important to provide advice to the obstetricians as to the urgency of delivery (which may reduce cardiovascular strain).

\section{Intra-operative Considerations}

The haemodynamic management of women around the time of birth is challenging given the gravid uterus, importance of blood pressure for uteroplacental blood flow and the potential for large volume blood loss and intravascular depletion. The use of neuroaxial techniques such as an epidural or spinal can cause sudden vasodilation, loss of preload and afterload causing a significant reduction in blood pressure. This can be compounded in a patient with PPCM as they lack the ability to compensate with an increase in contractility. Early epidurals during the first and second stage of labour may prove useful in PPCM as it allows good neuroaxial blockade to be established over a longer period of 
time, with incremental boluses. Epidurals may also prove helpful in reducing fatigue in the mother through better pain control.

Unless there is an emergency caesarean section, it would be prudent to have an arterial line placed prior to top up of an epidural or giving a spinal. If patients have severe decompensated heart failure, neuroaxial techniques may need to be avoided altogether, and a general anaesthetic employed. There is no evidence that one technique is better than another, and so the opinion of a senior anaesthetist should be sought. Wang et al recently published a case report of the anaesthetic management of PPCM where a 26 year old pregnant lady at 39 weeks gestation presented in florid pulmonary oedema and foetal distress. In that case they elected to perform a general anaesthetic and midway through the caesarean section the patient's oxygen requirements increased to $100 \%$ and had pink frothy fluid coming up from the endotracheal tube. The choice for general anaesthetic (over neuroaxial block) allowed them to manage the respiratory failure effectively whilst the caesarean section was completed [18].

As an interesting aside, they induced the patient with remifentanil and Propofol and maintained anaesthesia with combination of Propofol and remifentanil infusions. This is a slightly different technique to using inhalational anaesthetics for maintenance, and the use of remifentanil may have reduced the patient's ability to mount a tachycardia to maintain cardiac output. A case report by Kaufman et al highlights the importance of considering a differential for hypoxia in these patients. They describe a case of peripartum cardiomyopathy with concurrent pulmonary thromboembolism [19]. A combination of vasopressors and inotropes may necessitate placement of a central line. Care should be taken with rapid transfusion of blood or fluids. This may cause overdistention of myocardium with subsequent decompensation of heart failure. As such rapid blood tranfusers should generally be avoided and blood transfused judiciously with vasopressors to maintain blood pressure.

Acidosis, hypoxia and anaemia should be avoided as these can all contribute to myocardial dysfunction. In the operative setting this requires very close monitoring of blood loss and regular weighing of swabs to have an accurate input-output chart. Excess crystalloid resuscitation should also be avoided as this can lead to excess third space redistribution of fluids, potentially worsening pulmonary oedema. Patients with PPCM are at higher risk of developing arrhythmias which may need pharmacological or electrical intervention. Negative inotropic drugs such as beta blockers should be used with caution. Drugs such as digoxin and amiodarone maybe better options. Calcium channel blocker should also be used with caution due to their potential tocolytic effects.

\section{Post-operative considerations}

All patients with PPCM should be managed in an HDU environment immediately after delivery. If the patients present in the post-partum phase, they should be assessed clinically as to whether they would benefit from critical care level nursing and monitoring. This requires a senior review by a cardiology, general medical or critical care doctor. In the acute post-delivery stage these patients should ideally have invasive blood pressure monitoring with initially hourly followed by 4 to 6 hourly blood gasses for at least 24 hours. They should have active management of their third stage (irrespective of mode of delivery as they cannot tolerate large blood losses). It is vital that they have an accurate fluid chart with documentation of input and output. Daily weighing's of the mother may also be helpful to give an idea of fluid accumulation. Regular fluid status should be accessed through a combination of clinical examination, biochemical markers such as urea and creatinine and packed cell volume and advanced monitoring such as echocardiography or cardiac output monitoring and stroke volume variation (in the ventilated patient).

There is no evidence for which inotropic agent is most helpful but the options would include a phosphodiesterase inhibitor such as milrinone or a beta agonist such as dobutamine. Theoretically a calcium channel sensitizer such as levosimendan may be particularly useful for its ease of administration (a single infusion over 24 hours) which has been shown to have a prolonged effect of weeks up to months. This may theoretically allow quicker discharge from hospital and help bonding with the baby as the patient does not need to have a continuous infusion for days. There have been no randomised controlled trials into the most effective inotrope in the group of patients at present. These patients should have regular follow up with cardiologists whilst in hospital and be seen as outpatients for continual assessment of cardiac function.

\section{Conclusion}

PPCM is a rare condition that requires experienced clinicians to manage effectively. The anaesthetic considerations are based around maintaining a normal physiology in terms of acid-base balance, hypoxia and anaemia. The intravascular volume needs careful managed to ensure adequate preload for maintenance of cardiac output. Equally excess fluid replacement should be avoided, especially with crystalloids, as it may cause decompensation of heart failure or additional extravascular fluid shifts, exacerbating pulmonary oedema. A careful continuous assessment of intravascular volume is vital in the preoperative, intraoperative and postoperative period to ensure safe management of these patients. The prognosis is good for these patients with half returning to a normal systolic function. Treatment modalities such as bromocriptine should be considered in refractory cases, although the evidence for its use is still being developed. There is no established anaesthetic treatment protocol for these patients. The decision of neuroaxial block versus a general anaesthetic has to be judged on a patient by patient basis. Depending on the level of physiological reserve and the urgency of the delivery of the baby an epidural with incremental top ups may be a better option with invasive blood pressure monitoring and potentially a central line for vasopressor and inotropic drug administration. Advanced haemodynamic monitoring with cardiac output monitors may 
help guide fluid replacement, vasopressor use and deciding when to instigate inotropic drugs (as well as helping to titrate doses).

\section{Acknowledgement}

None.

\section{Conflicts of Interest}

None to declare.

\section{References}

1. Gupta D, Wenger NK (2018) Peripartum cardiomyopathy: Status 2018. Clin Cardiol 41(2): 217-219.

2. Sliwa K, Hilfiker Kleiner D, Petrie MC, Mebazaa A, Pieske B, et al. (2010) Current state of knowledge on aetiology, diagnosis, management, and therapy of peripartum cardiomyopathy: a position statement from the Heart Failure Association of the European Society of Cardiology Working Group on peripartum cardiomyopathy. Eur J Heart Fail 12(8): 767-778.

3. Coyle JL, Jensen L, Sobey A (2012) American College of Cardiology Foundation; American Heart Association. Peripartum cardiomyopathy: review and practice guidelines. Am J Crit Care 21(2): 89-98.

4. Tidswell M (2004) Peripartum cardiomyopathy. Crit Care Clin 20(2): 777-788.

5. Sliwa K, Mebazaa A, Hilfiker KD, Petrie MC, Maggioni AP, et al. (2017) Clinical characteristics of patients from the worldwide registry on peripartum cardiomyopathy (PPCM): EURObservational Research Programme in conjunction with the Heart Failure Association of the European Society of Cardiology Study Group on PPCM. Eur J Heart Fail 19(9): 1131-1141.

6. Elkayam U, Akhter MW, Singh H, Khan S, Bitar F, et al. (2005) Pregnancy-associated cardiomyopathy: clinical characteristics and a comparison between early and late presentation. Circulation 111(16): 2050-2055.

7. Gunderson EP, Croen LA, Chiang V, Yoshida CK, Walton D, et al. (2011) Epidemiology of peripartum cardiomyopathy: incidence, predictors, and outcomes. Obstet Gynecol 118(3): 583-591.

8. Hilfiker Kleiner D, Haghikia A, Nonhoff J, Bauersachs J (2015) Peripartum cardiomyopathy: current management and future perspectives. Eur Heart J 36(18): 1090-1097.
9. Ansari AA, Fett JD, Carraway RE, Mayne AE, Onlamoon N, et al. (2002) Autoimmune mechanisms as the basis for human peripartum cardiomyopathy. Clin Rev Allergy Immunol 23(3): 301-324.

10. Lapaire O, Hosli I, Zanetti DR, Huang D, Jaeggi C, et al. (2007) Impact of fetal-maternal microchimerism on women's health-a review. J Matern Fetal Neonatal Med 20(1): 1-5.

11. Patten IS, Rana S, Shahul S, Glenn C. Rowe, Cholsoon Jang, et al. (2012) Cardiac angiogenic imbalance leads to peripartum cardiomyopathy. Nature 485: 333-338.

12. Hunt SA, Abraham WT, Chin MH, Feldman AM, Francis GS, et al. (2009) Focused update incorporated into the ACC/AHA 2005 Guidelines for the Diagnosis and Management of Heart Failure in Adults A Report of the American College of Cardiology Foundation/American Heart Association Task Force on Practice Guidelines Developed in Collaboration With the International Society for Heart and Lung Transplantation. J Am Coll Cardiol 53(15): e1-e90.

13. Lata I, Gupta R, Sahu S, Singh H (2009) Emergency management of decompensated peripartum cardiomyopathy. J Emerg Trauma Shock. 2(2): 124-128.

14. Hilfiker KD, Haghikia A, Berliner D, Vogel CJ, Schwab J, et al. Bromocriptine for the treatment of peripartum cardiomyopathy: a multicentre randomized study. Eur Heart J 38(35): 2671-2679.

15. Safirstein JG, Ro AS, Grandhi S, Wang L, Fett JD, et al. (2012) Predictors of left ventricular recovery in a cohort of peripartum cardiomyopathy patients recruited via the internet. Int J Cardiol 154(1): 27-31.

16. Heart Failure Society of America (2006) Executive summary: HFSA 2006 Comprehensive Heart Failure Practice Guideline. J Card Fail. 12(1): $10-38$

17. Biteker M, Ilhan E, Biteker G, Duman D, Bozkurt B (2012) Delayed recovery in peripartum cardiomyopathy: an indication for long-term follow-up and sustained therapy. Eur J Heart Fail 14(8): 895-890.

18. Wang J, Han Z, Zou Y, Wang N (2017) Anaesthetic management of a pregnant patient with acute pulmonary oedema and peripartum cardiomyopathy: A case report. Anaesth J E M (2017) 24(2): 155-158.

19. Kaufman I, Bondy R, Benjamin A (2003) Peripartum cardiomyopathy and thromboembolism; anesthetic management and clinical course of an obese, diabetic patient. Can J Anaesth 50(2): 161-165.

\section{Your next submission with Juniper Publishers will reach you the below assets}

- Quality Editorial service

- Swift Peer Review

- Reprints availability

- E-prints Service

- Manuscript Podcast for convenient understanding

- Global attainment for your research

- Manuscript accessibility in different formats

( Pdf, E-pub, Full Text, Audio)

- Unceasing customer service

Track the below URL for one-step submission https://juniperpublishers.com/online-submission.php 